\title{
Drosophila Pif1A is essential for spermatogenesis and is the homolog of human CCDC157, a gene associated with idiopathic NOA
}

\author{
Xin Yuan ${ }^{1,2}$, Huimei Zheng ${ }^{1,2}$, Yang Su', Pengfei Guo ${ }^{1,2}$, Xiao Zhang ${ }^{1,2}$, Qiang Zhao ${ }^{2}$, Wanzhong Ge ${ }^{1,2}$, Chen Li \\ Yongmei $\mathrm{Xi}^{1,2}$ and Xiaohang Yang ${ }^{1,2,3}$
}

\begin{abstract}
The dynamic process of spermatogenesis shows little variation between invertebrate models such as Drosophila, and vertebrate models such as mice and rats. In each case, germ stem cells undergo mitotic division to proliferate and then continue, via meiosis, through various stages of elongation and individualization from spermatogonia to spermatid to finally to form mature sperm. Mature sperm are then stored in the seminal vesicles for fertilization. Errors in any of these stages can lead to male infertility. Here, we identify that Drosophila Pif1A acts as a key regulator for sperm individualization. Loss of Pif1A leads to male sterility associated with irregular individualization complex and empty seminal vesicles without mature sperm. Pif1A is highly expressed in the testes of mated male adult flies and the Pif1A protein is expressed at a higher level in male than in female flies. Pif1A is homologous to mammalian coiled-coil domain-containing protein 157 (CCDC157), which is also enriched in the testes of humans and mice. Human CCDC157, with unknown function, was identified to be downregulated in men with idiopathic non-obstructive azoospermia (NOA). We map the function of Drosophila Pif1A during spermatogenesis, showing that Pif1A is essential for spermatide individualization and involved in the regulation of the lipid metabolism genes. Our findings might be applicable for studying the function of CCDC157 in spermatogenesis and other aspects of human male fertility.
\end{abstract}

\section{Introduction}

Spermatogenesis is a highly conserved process, not differing appreciably between any animal model, from Drosophila in insects to mice and rats in mammals ${ }^{1}$. Each developmental stage is precisely controlled by intrinsic signals. Human male infertility results from abnormal

Correspondence: Chen Li (chenli2012@zju.edu.cn) or Yongmei Xi (xyyongm@zju.edu.cn)

${ }^{1}$ Division of Human Reproduction and Developmental Genetics, the Women's Hospital, Zhejiang University School of Medicine, Yuhangtang Road 866, 310012 Hangzhou, Zhejiang, China

${ }^{2}$ Institute of Genetics and Department of Genetics, Zhejiang University School of Medicine, Yuhangtang Road 866, 310012 Hangzhou, Zhejiang, China

Full list of author information is available at the end of the article.

These authors contributed equally: Xin Yuan, Huimei Zheng

Edited by E. Baehrecke spermatogenesis and is mostly due to chromosomal alterations, $\mathrm{Y}$ chromosome microdeletions, and related gene mutations ${ }^{2}$. For many of these, the detailed mechanisms are often technically inaccessible and remain obscure. In Drosophila, the testes are easy to dissect and the morphological changes during each stage of spermatogenesis can be easily visualized ${ }^{3}$. Drosophila is, therefore, well established as an excellent model for the study of spermatogenesis ${ }^{4}$. Recently, a large-scale RNAi screen in Drosophila testes has been conducted to analyze the genes required for germline stem cell (GSC) maintenance or differentiation ${ }^{5}$. Many of these genes were noted as those involved in key steps of protein synthesis and degradation relating to GSC homeostasis ${ }^{5-7}$, while others were identified as key regulators of the dynamic process of

\section{(c) The Author(s) 2019}

(c) (i) Open Access This article is licensed under a Creative Commons Attribution 4.0 International License, which permits use, sharing, adaptation, distribution and reproduction cc) in any medium or format, as long as you give appropriate credit to the original author(s) and the source, provide a link to the Creative Commons license, and indicate if changes were made. The images or other third party material in this article are included in the article's Creative Commons license, unless indicated otherwise in a credit line to the material. If material is not included in the article's Creative Commons license and your intended use is not permitted by statutory regulation or exceeds the permitted use, you will need to obtain permission directly from the copyright holder. To view a copy of this license, visit http://creativecommons.org/licenses/by/4.0/. 
sperm morphogenesis ${ }^{8,9}$. However, the molecular links between different stages of spermatogenesis remains largely unidentified. The combination of various genetic techniques applied to Drosophila, such as CRISPR/Cas $9^{10}$ and the Gal4/ UAS transgenic system ${ }^{11}$, have enabled the convenient mapping and prediction of functional genes which facilitate, for example, the identification of genes that might cause infertility due to deficiencies in a specific developmental stages or in certain type of cells.

To gain insight into the molecular basis for male sterility, we made use of CRISPR/Cas9 to produce mutations of the genes shown to be highly expressed in Drosophila testis. A gene of unknown function, PFTAIRE interacting factor $1 \mathrm{~A}(\text { Piff } A)^{12}$, was of particular interest. The female mutants of Pif1A displayed normal fertility but the males were infertile. Pif1A is the only homolog of the mammalian coiled-coil domain-containing protein 157 (CCDC157) family (www.pantherdb.org). Pif1A has been noted to exist at a high transcriptional expression level in the testes of mated male flies (www.flybase.org) and the Pif1A protein is expressed at a higher level in male than in female flies ${ }^{12}$. Notably, CCDC157 has also been observed as highly expressed in the testes of humans and mice, and to be downregulated in men with idiopathic nonobstructive azoospermia (NOA) ${ }^{13,14}$. Here, we map the function of Pif1A during Drosophila spermatogenesis, which might be informative for human CCDC157.

Male Drosophila adults contain a pair of testes, each testis is a coiled tube with a closed apical end and a basal end that connects to the seminal vesicle. At the apical end there are approximately 8-12 GSCs. Spermatogenesis takes place within individual units known as cysts $^{15}$, where GSCs divide asymmetrically in the cysts to give rise to spermatogonial cells. Each GSC is flanked by two somatic cyst stem cells (CySCs) that eventually differentiate into a head cyst cell and a tail cyst cell, analogous to mammalian Sertoli cells ${ }^{16,17}$. Spermatogonia then go through four mitotic divisions, generating 16 primary spermatocytes that further undergo two meiotic divisions to yield a group of 64 syncytial haploid spermatids. Round spermatids are inter-connected by abundant cytoplasmic bridges ${ }^{15,18}$. During post-meiotic spermatid differentiation, syncytial cysts of 64 haploid spermatids undergo synchronous differentiation. Numerous changes occur at a subcellular level including formation of flagellar axonemes and acrosomes, remodeling of mitochondria and nuclei, and the polarization of elongating cysts and the plasma membrane 6 . The fully elongated syncytium of 64 spermatids undergoes a membrane remodeling process known as individualization ${ }^{18,19}$.

Individualization begins with the formation of investment cones around each of the 64 needle-shaped nuclei ${ }^{16,18}$. These investment cones assemble into a macroscopic structure referred to as the individualization complex (IC), from which the cyst membrane is remodeled and intercellular bridges are resolved to encase each sperm cell in its own plasma membrane ${ }^{18,19}$. The IC moves processively from the heads to the tips of the tails along the spermatid bundle ${ }^{20}$. During this process unneeded organelles, mitochondrial DNA, and cytoplasmic components are stripped away, forming the observable dilation of the cyst known as the "cystic bulge". The cystic bulge is then detached at the tip of the tail where it becomes known as the "waste bag"21.

Individualization results in the formation of individual sperm. The structure of the actin cones and their cohort movement is critical for spermatid individualization. Cytoskeletal regulators, such as myosin V, myosin VI, cortactin, and Arp $2 / 3$ complex, have been identified to influence the formation of actin cones and the synchronous movement of the $\mathrm{IC}^{22,23}$. In myosin $\mathrm{V}$ mutants, fewer actin cones were able to form ${ }^{24}$. Myosin VI acts to stabilize the actin cones and the Arp $2 / 3$ complex is required for the formation of the actin meshwork ${ }^{25-27}$. Cortactin co-localizes with Arp $2 / 3$ complex and myosin VI at the leading edge of the actin cone during IC movement ${ }^{28}$.

The individualization process has also been defined as a caspase-dependent apoptosis-like event, requiring functional proteasomes and membrane trafficking ${ }^{16,21}$. Multiple Drosophila caspases and caspase regulators participate in this process. Mutation of any of these genes leads to individualization defects ${ }^{9}$. During complex membrane remodeling, lipids including phospholipids, sphingolipid and cholesterol, act as essential membrane structural components and also play critical roles. Lipid regulators including Drosophila nessy (nes), oysgedart (oys), noa, oxysterol binding protein (osbp), fan, npc1 have been identified ${ }^{29-32}$, although the role of most of their protein products remains obscure. The mutation of $o s b p$, for example, leads to abnormal sterols trafficking and distribution and in corresponding male infertility ${ }^{30}$. The regulation mechanism of OSBP remains unclear.

In this study, we have identified that PiflA is essential for spermatid individualization and might act to regulate lipid metabolism genes during spermatogenesis. Loss of Pif1A results in the downregulation of several lipid regulators and in a phenotype that genetically mirrors that of the loss of $o s b p$. By dissecting the testes and conducting immunofluorescence staining, we found that Pif1A mutants showed normal GSCs divisions and spermatid elongation but displayed disorganized IC and irregular IC movement. Correspondingly, no mature sperm could be observed in their seminal vesicles. The duplication of the Pif1A genome could partially rescue this phenotype. We show that Pif1A plays a critical role during spermatogenesis and thus is essential for male fertility. Our findings might be applicable for studying the function of 
CCDC157 in spermatogenesis and in other aspects of human male fertility.

\section{Results \\ Identification of Pif1A, homologous to human CCDC157, as a novel regulator for male fertility}

To identify novel regulators in spermatogenesis, using CRISPR/Cas9, we generated a series of mutants of the various genes that had high transcript expression levels in the testis of adult male (www.flybase.org). We focused on Pif $1 A$ and identified that one of the mutations, Pif $1 A^{1}$, resulted in compromised male fertility but left female fertility unaffected. Expression of Pif1A mRNA in WT adult testis is clarified by whole-mount in situ hybridization (Fig. 1a). The gRNA for Pif1A was designed to recognize a 19-nt target sequence in a position where it always plays as an exon role among five transcripts and acts to direct Cas9-mediated cleavage of both DNA strands within the target site. PiflA ${ }^{1}$ is a frame shift nonsense mutant allele with 2 bp deletion (Fig. 1b) leading to an earlier stop codon 12 amino acids from the deletion position. We analyzed the transcription levels of Pif1 $A^{1}$ in homozygous adult flies and trans heterozygous adult animals from the $P i f 1 A^{1}$ allele and a deficiency line uncovering Pif1A (Pif1A $1 / D F)$ by quantitative PCR (qPCR). The mRNA levels were significantly reduced in

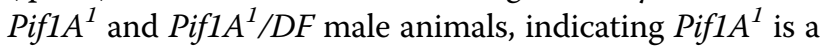
loss-of-function mutation (Fig. 1c). This is the first loss of function allele for Pif1A, in contrast to the numerous other alleles listed in Flybase. Fertility test showed that WT females crossed with Pif $1 A^{1}$ or Pif $1 A^{1} / D F$ males laid similar number of eggs as did those crossed with the WT males (Table S1), but the percentage of hatched embryos derived from Pif1A mutant males was zero (Fig. 1d). In addition, we carried out a genomic rescue experiment through a transgenic fly BL42670 (www.flybase.org). The duplication of the Pif1A genome was able to partially restore the male reproductive capacity (Fig. 1d).

Sequence analysis identified that Drosophila Pif1A is homologous to the mammalian CCDC157, with conserved SMC_N super family domain (Fig. 2a, a', a'). The Pif1A protein has a $20 \%$ identity and $34 \%$ similarity to human CCDC157 and the predicted three-dimensional (3D) structure of Pif1A is similar to that of human CCDC157 (Fig. 2a"). We measured the mRNA levels of CCDC157 in various mouse tissues. The CCDC157 transcript was highly enriched in testes compared with the other tissues (Fig. 2b). We then performed a quantitative transcriptomics analysis through open databases ${ }^{33}$ and found that human CCDC157 was also highly enriched in the testes (Fig. 2c). The expression levels of CCDC157 in men with normal spermatogenesis were significantly higher than the patients with idiopathic $\operatorname{NOA}^{13}$ (Fig. 2d). We further analyzed recently published single-cell RNA- sequencing data (GEO:GSE106487) underlying a hierarchical model of human testicular cells ${ }^{14}$. In addition to their findings on several stage-specific marker genes of testicular germ cells, such as HMGA1, PIWIL4, TEX29, SCML1, and CCDC112 $2^{14}$, our analysis highlights that CCDC157 is also highly expressed in spermatid stages but rarely expressed in spermatogonia and spermatocyte stages (Fig. S1A). Totally, 2854 individual testicular cells from donors with normal spermatogenesis showed significant higher expression of CCDC157 than 174 testicular cells from one NOA donor (Fig. S1B).

\section{Pif1A mutants displayed normal GSC divisions and spermatid elongation, but an empty seminal vesicle}

We firstly examined the gonads of homozygous ${\text { Pif } 1 A^{1}}^{1}$ adult fly. The morphology of the reproductive system, including accessory gland and ejaculatory duct, seemed normal in Pifl $1 A^{1}$ compared to those of control animals (Fig. 3a, b). However, the seminal vesicle (SV) from the

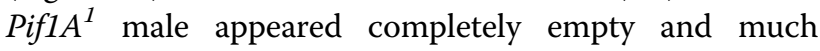
smaller (Fig. 3a', b). Squashes of SVs confirmed the presence of motile sperm in the control animals as contrasted to their complete absence in Pif $1 A^{1}$ mutants.

We then examined the spermatogenesis processes, beginning with the evaluation of germ cell division. We constructed nos-GAL4, mainly expressed in early germ cells including GSCs but with faded expression in spermatocytes $^{34}$, driving UAS-CD8GFP in Pif1A mutant background. By dissecting the gonad and analyzing GFP patterns, no obvious differences were found between the mutants and control animals (Fig. 3c', d). Similarly, the GFP patterns drove by bam-GAL4, mainly expressed in spermatogonia, or tj-Gal4, expressed in CySCs and cyst cells ${ }^{5}$, also appeared to be unaffected in PiflA mutants (Fig. 3c-f). The Pif $1 A^{1}$ testes also showed normal intercellular connections ${ }^{35}$ (Fig. 3g, h). The primary spermatocyte cysts at the 16-cell stage and spermatid cysts at the onion stage (64-cell) are identical to those in the WT (Fig. S3A). These observations suggest that mutation of Pif1A has no effect on germ cell divisions.

We next explored the spermatid elongation by constructing an $\beta$ Tub85D-GFP as a spermatocyte-specific marker $^{36}$, and a Don Juan-GFP, a marker for late spermiogenesis ${ }^{33,37,38}$ into the Pifl $A^{1}$ background animals. The Don Juan protein is produced in the giant sperm tail's mitochondria and peaks once the nuclei have condensed and persists into mature sperm, but is not found in spermatids that are still undergoing elongation $^{33,37,38}$. Observing GFP markers, elongated sperm tails showed up as equally normal in both WT and Pif $1 A^{1}$ animals (Fig. 3i, l). However, the fluorescence was not detectable in the seminal vesicles of Pif $1 A^{1}$ mutants (Fig. 3j, 1), but was present in that of WT animals (Fig. 3i, k). These results indicate that mutation of Pif1A 
A
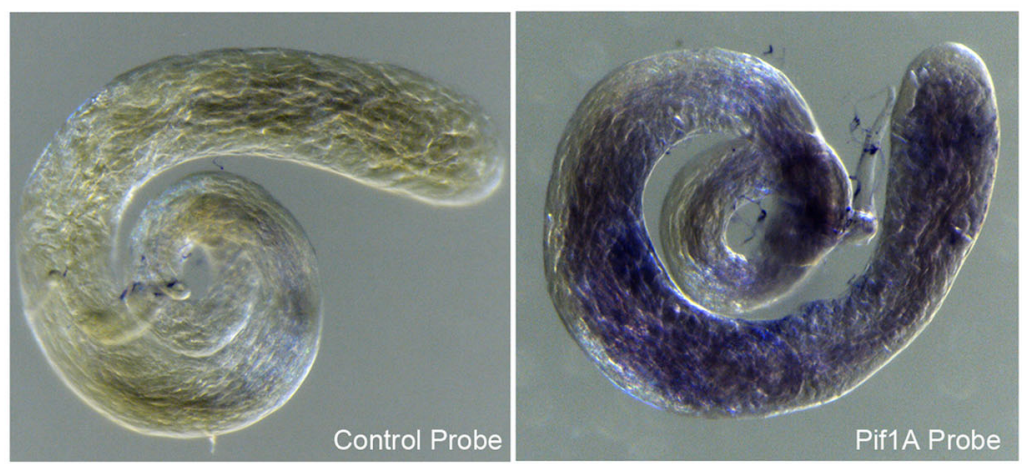

B

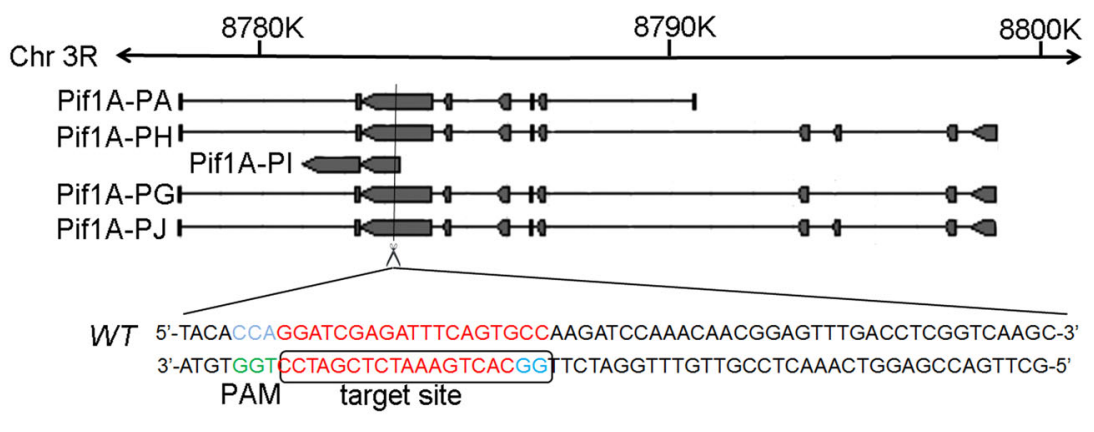

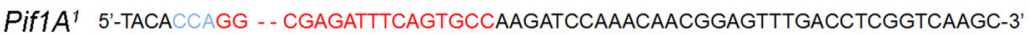

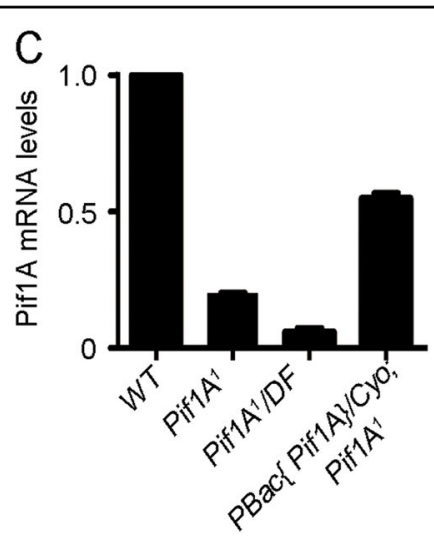

D

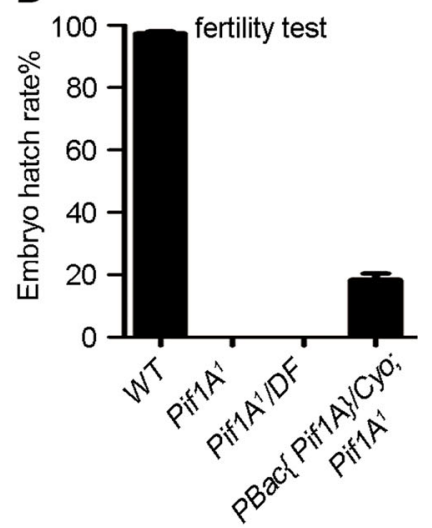

Fig. 1 Loss-of-function of Pif1A results in male-sterile. a Whole-mount in situ hybridization for control probe (left) and Pif1A probe (right) on testis of adult flies. $\mathbf{b}$ Schematic representation of the Pif1A locus. Pif1A is located on chromosome 3R at 85B1-85B1 on the cytogenetic map. Filled tetragonums indicate exons; the straight lines between filled tetragonums indicate introns. Pifl $A^{\prime}$ is a frame shift mutant allele with 2 bp deletion. $\mathbf{c}$ Real-time PCR analysis of Pif1A transcriptional levels of wild-type animals, Pif1A mutants, Pif1 $A^{\top}$ over deficiency (Pif1 $\left.A^{\top} / D F\right)$ animals and the duplication of Pif1A genomic DNA in the background of Pif1 $A^{7}$ (Pif1A-GFP.FPTB/Cyo;Pif1 $A^{1} /$ PiflA $^{1}$ ). $\mathbf{d}$ Male fertility test. $y$-axis represented embryo hatch rate. The male fertility was zero in Pif1 $1 A^{\prime}$ mutants and Pif1 $1 A^{\prime} / D F$ animals and partially restored in the duplication of Pif1A genomic DNA in the background of Pif1 $A^{\prime}$ animals

may leads to a post-meiotic defect after spermatid elongation.

Loss of Pif1A results in lack of cystic bulges and waste bags

The final stage of spermatid differentiation involves the removal of bulk cytoplasm. The DrICE is cleaved and activated throughout the length of elongated spermatids ${ }^{9}$. In WT testis, the elongated spermatids, cystic bulges (CBs) and waste bags (WBs) could be visualized with antibodies specific for activated DrICE (Fig. 4a). In Pif1 $A^{1}$ mutants, no CBs or WBs appeared, although DrICEpositive signals were partially detectable (Fig. 4b, c). We then extracted the protein and total RNA from the testes of Pif1 $A^{1}$ and WT animals. Western blot showed that the levels of active DrICE were not decreased (Fig. 4d, d') and the transcript levels of two other genes related to caspase activity, Ark and Dronc were also not reduced in the mutants (Fig. 4e). These observations demonstrate that the lack of CBs and WBs in the testes of Pif1A mutants is not due to downregulated caspase activity.

\section{Pif1 $A$ is required for the spermatid individualization process}

We next investigated the spermatid individualization process by visualizing the F-actin component of IC with fluorescent-conjugated phalloidin staining. In WT testes, the IC was correctly assembled around the nuclei and traveled down the cysts (Fig. 5a and Movie S1), and the WBs were evident (Fig. 5b, Movie S3). However, in the Pif $1 A^{1}$ mutants, the IC movement was limited and disorganized (Fig. 5c, d and Movie S2), where only a few actin cones moved a short distance from the nuclei (Fig. 5e, h). The orientation of the actin cones had been reversed in Pif1 $A^{1}$ mutant (Fig. 5i, j), with no moving CB in the cyst (Movie S4). The barbed end of the actin cones remains close to the nuclei in the WT testis ${ }^{39-41}$ (Fig. 5f), 


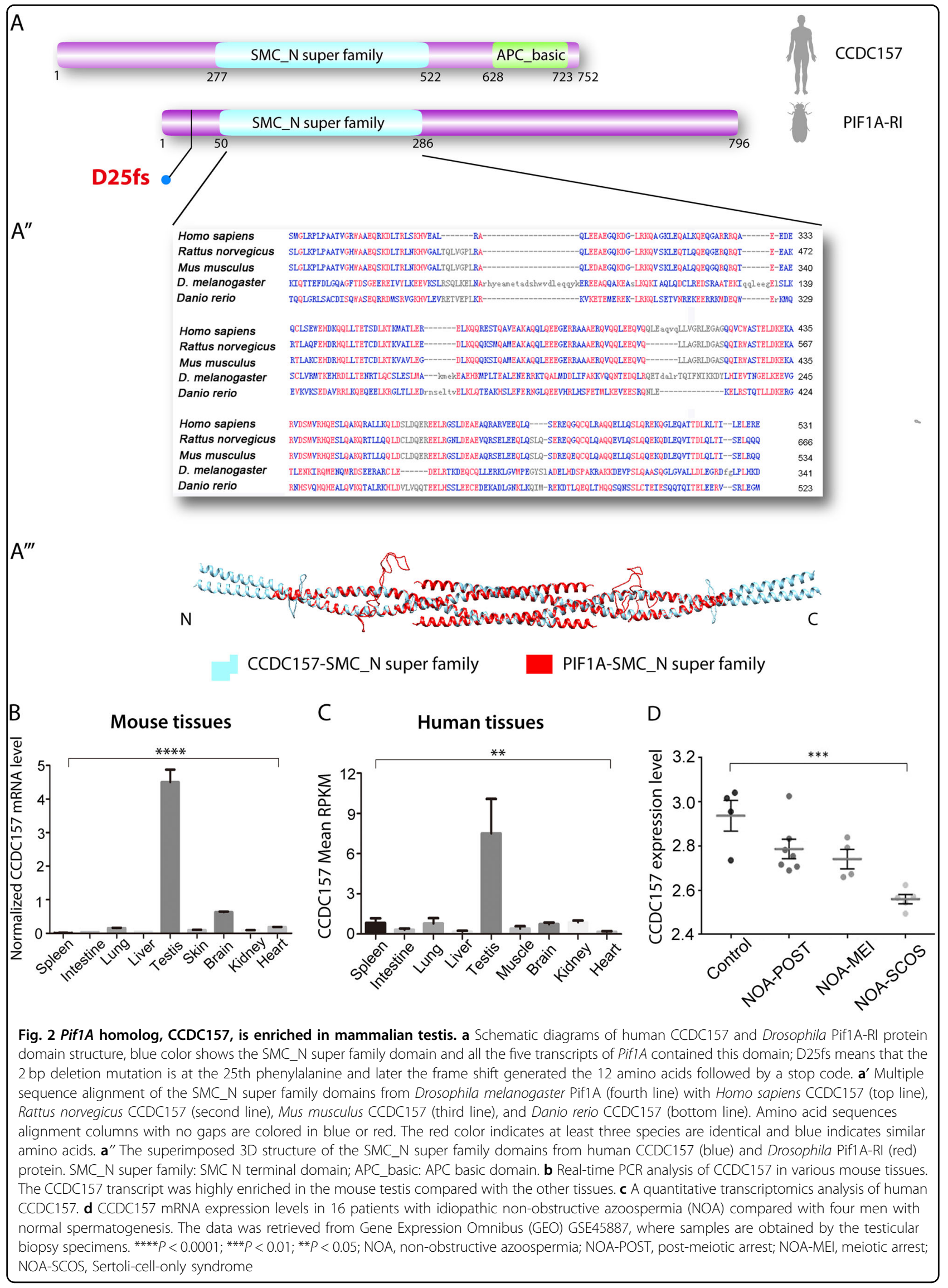


whereas, in Pif $1 A^{1}$ testes, the barbed end presented on the reverse side from the nuclei (Fig. 5i, j). Similar ICs formed at the beginning and almost zero progressed uniformly, in which the total number of ICs per testis was significantly reduced in the mutants (Fig. 5e).

We also examined the cyst during the individualization process using transmission electron microscopy (TEM). In the WT, very little cytoplasm was left in individualized sperm and that each axoneme/mitochondria pair was tightly surrounded by a clear integral plasma membrane (Fig. 5k) and the axonemal microtubules showed a $9+2$ organization (Fig. $5 \mathrm{~m}$ ). However, in the Pif $1 A^{1}$ mutants, only a few axoneme/mitochondria pairs occurred, and many sperm tails were enclosed within the same membrane and surrounded by a large amount of cytoplasm (Fig. 5l), although the organization of axonemal microtubules in Pif $1 A^{1}$ mutant spermatids seemed normal (Fig. 5n). The nebenkern of Pif $1 A^{1}$ is smaller than that of the WT (Fig. 5m, $\mathrm{n}$ and Fig. S3B). The mRNA levels of the related nuclear genes ${ }^{42-48}$ indicated that none but merlin, that has been previously demonstrated to be important for the formation and modification of the nebenkern ${ }^{47,48}$, was significantly downregulated (Fig. S3E). These data together suggest that Pif1A might be required for proper aligning and synchronously movement of IC.

\section{Individualization failure is not caused by a problem in nuclear shaping}

During nuclear condensation, somatic histones had been replaced by protamines. In Drosophila, the mammalian homolog of the protamines and transition proteins were identified as ProtamineA/Protamine $B$ and Tpl94D ${ }^{49,50}$. We constructed ProtB-eGFP in PiflA ${ }^{1}$ mutants background and evaluated the GFP patterns. ProtB-eGFP did not express in young elongating nuclei and early canoe stage spermatids but begun expression from the late canoe stage spermatids onwards ${ }^{50}$. The GFP signals in late canoe stage spermatids were detected in both Pif1 $A^{1}$ and WT animals (Fig. 6a', b). After individualization, the ProtB-eGFP became more condensed and corresponded to and overlapped the nuclei (Fig. 6a). GFP signals were clearly detectable in mature sperm in the testis lumen in WT (Fig. 6a', a'). In Pif1 $A^{1}$ mutants, the intensive ProtB-eGFP overlapping the needle-shaped nuclei were less defined (Fig. 6b), indicating they had begun to be progressively eliminated.

Spermatid nuclei serve as a physical scaffolding for the assembly of the individualization complex ${ }^{18,48}$. We checked the nuclear shaping during spermiogenesis. In morphology, we could observe the round nuclei, canoeshaped nuclei and typical needle-shaped nucleus in the testes of both WT animals and Pif1A ${ }^{1}$ (Fig. 6c, d'). The transcription levels of the nuclear shaping related genes $^{37,51-55}$ remained unaltered in Pif1A ${ }^{1}$ (Fig. S4A).
However, comparing to that in the WT, fully compacted nuclei are tightened after removing unneeded cytoplasmic components (Fig. 6a, a", c, c'), the needle-like nuclei in Pif $1 A^{1}$ are toward to lose (Fig. $6 \mathrm{~b}-\mathrm{d}^{\prime \prime}$ ). Immunostaining with Caspase 3 in the cyst assembly towards to the terminal epithelium region, where the mature sperms are released into the testis lumen and then pass into the seminal vesicle, the signal was not detectable in the WT (Fig. S6A), but obvious in Pif1 $1 A^{1}$ mutants (Fig. S6B). This indicated that up-regulated Caspase 3 and cells death occurred at the end of spermatogenesis.

\section{Lipid metabolism genes are downregulated in the testis in absence of Pif1A}

The observations of irregular pattern and reduced number of ICs in PiflA ${ }^{1}$ are similar to phenotypes caused by downregulation of myosin $\mathrm{VI}^{25,28,56}$. We sought to analyze the transcription levels of myosin VI, that acts in a structural capacity in the $\mathrm{IC}^{25}$, and cortactin and Arp $2 / 3$ complex, which play critical roles in regulating actin polymerization $^{22,27,57}$. Real-time PCR results revealed no significant changes in transcription levels of actin-related genes in the testes of PiflA ${ }^{1}$ and WT (Fig. S4B). However, when we examined expression levels of a group of lipid metabolic genes, the mRNA levels of nes, oys, noa, osbp and fan were dramatically downregulated in the testes of Pifl $A^{1}$ mutants (Fig. 7e).

We finally examined the cellular localization of Pif1A protein by fusion proteins generated in our experiments. Both eGFP-Pif1A-RG and Pif1A-RI-Flag were detected mainly in the nucleus (Fig. $7 \mathrm{a}, \mathrm{b}^{\prime}$ ). Immunostaining with Eya antibody and Pif1A-RI-Flag correspondingly evidenced its spermatid-specific expression (Fig. S2). We tested Pif1A overexpression phenotype and observed that adult flies with overexpression of Pif1A-RG driven by Daughterless-Gal4 displayed unexpanded wings and that adult flies with overexpression of Pif1A-RI driven by a hsGal4 displayed abnormal dorsal bristles (Fig. 7c, d). For Pifl1A, both deletion and overexpression mutants resulted in similar phenotypes to those of OSBP, respectively, where OSBP had also been demonstrated genetically and physically to regulate the individualization process $^{30}$. These results suggest that Pif1A might act upstream of lipid metabolism genes, such as OSBP, to regulate individualization during Drosophila spermatogenesis.

\section{Discussion}

In this study, we have applied Drosophila tool kit with the UAS/GAL4 system and CRISPR/Cas9 methods to determine gene function in specific aspects of spermatogenesis, linking abnormal gene function with infertility. Pif1A, the homolog of human CCDC157 that has been shown to be downregulated in men with idiopathic NOA, has been confirmed here to be required for spermatid 

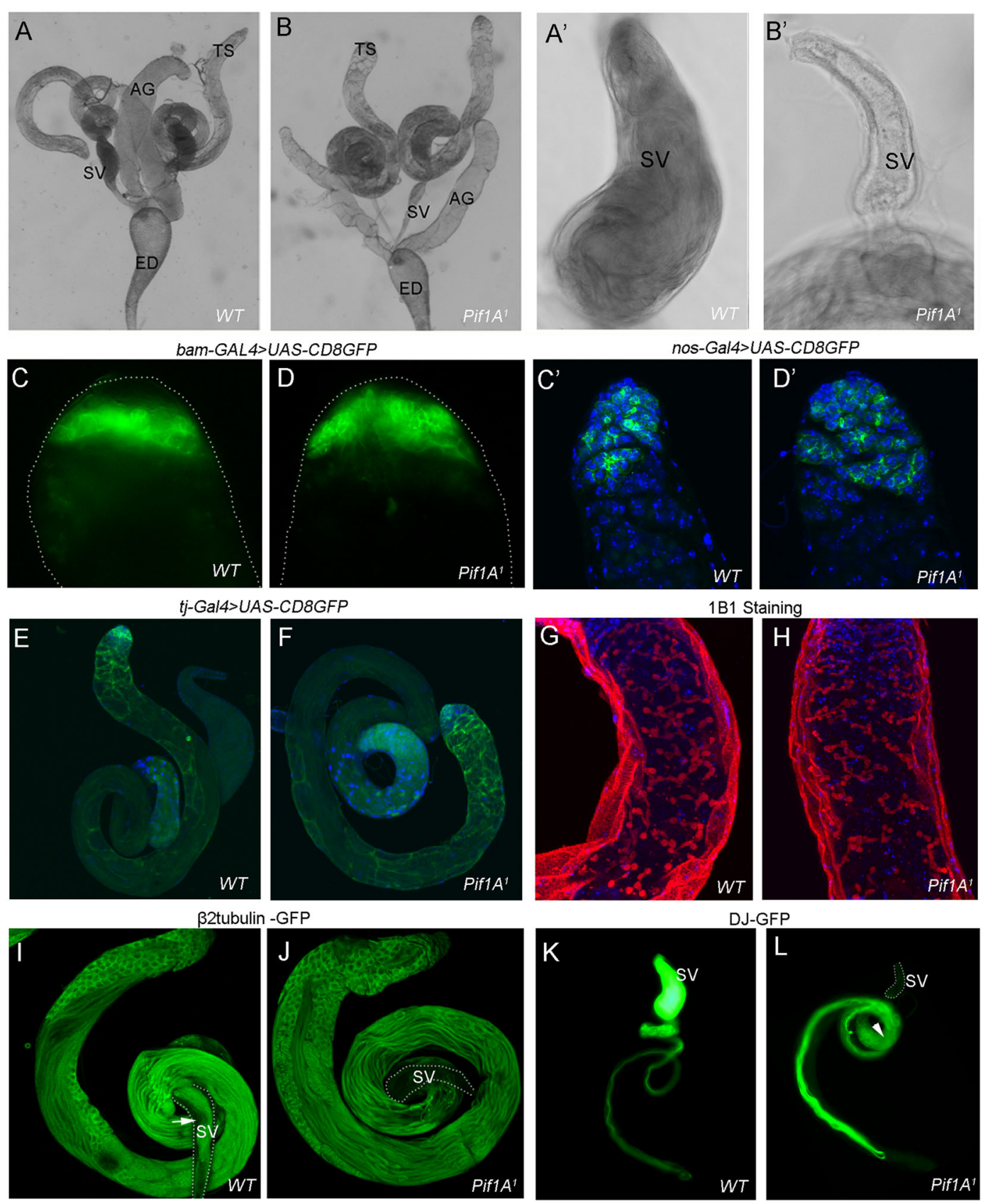

Fig. 3 Germ cell divisions and spermatid elongation in Pif1 $A^{1}$ mutant seems unaffected. Images of dissected male reproductive system from wild-type animals (a) and Piff $A^{1}$ mutants (b); TS, testis; SV, seminal vesicle; AG, accessory gland; ED, ejaculatory duct. The magnified image of SV from

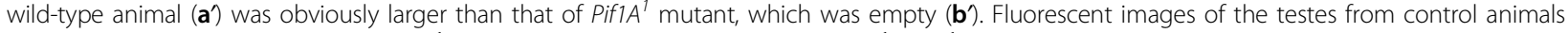
(bam-GAL4 > UAS-CD8GFP) (c) and Pif1 A'mutants (bam-GAL4 > UAS-CD8GFP; Pif1A'/Pif1A ${ }^{\prime}$ ) (d) showed that spermatogonia had divided normally.

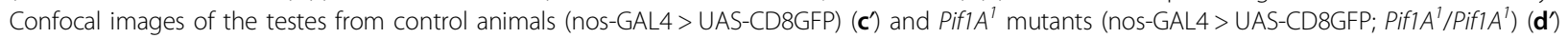
indicated that early germ cells were not affected in PiflA $A^{7}$ mutant (Green;GFP, blue; DAPI). Images of the testes from control animals (tj-Gal4 $>$ UASCD8GFP) and Pif1 $A^{7}$ mutants (tj-Gal4 > UAS-CD8GFP; Pif1 $A^{\top} / P_{i f 1} A^{\top}$ ) (f) suggested that CySCs and cyst cells were also not affected in Pif1 $A^{7}$ mutants. 1 B1 staining representing fusomes showed similar patterns in the testes of wild-type animals $(\mathbf{g})$ and Pif1 $A^{\prime}$ mutants (h). The testis-specific $\beta 2 t u b u l i n$ promoter drives GFP shows that elongated spermatid bundles existing in both wild-type (i) and Pif1 $A^{\prime}$ mutant (j), and seminal vesicles containing stored mature sperm (i, arrow) in wild-type animals but not in the Pif1 $A^{\top}$ mutants. Elongated sperm tails, marked with Don Juan-GFP (green), fill the seminal vesicle in wild-type testes $(\mathbf{k})$. In the Pifı $A^{\top}$ mutants, their elongated sperm tails are also shown in the testis but the seminal vesicle is empty (I) 

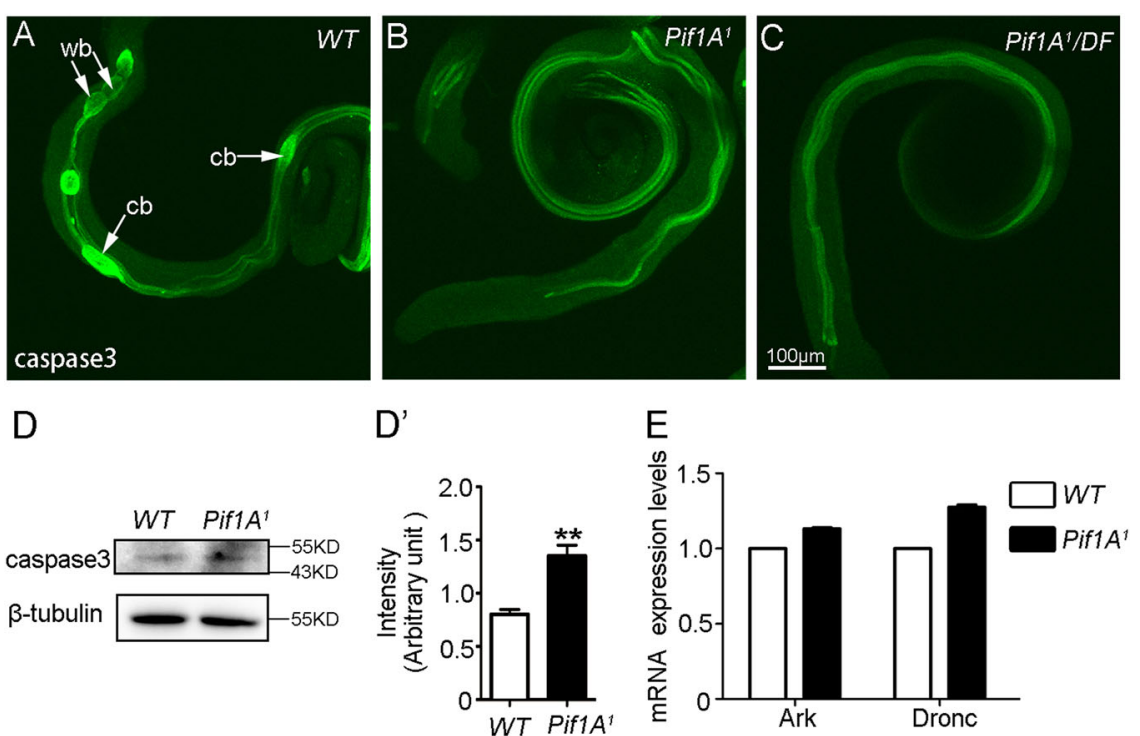

Fig. 4 Loss-of-function of Pif1A leads to lacking of cystic bulges and waste bags. a-c Testes of different genotypes were visualized with antibodies specific for caspase 3 (green). a Wild-type testis. Caspase 3 was present in multiple elongated cysts. Cystic bulges (cb) and waste bags (wb) are indicated by arrows; b, c Testes from Pif1 $A^{1}$ homozygote mutants and Pif1 $A^{1} /$ DF heterozygotes, respectively; cystic bulges and waste bags were hard to find. $\mathbf{d}$ Western blot detected by anti-caspase 3 and anti-tubulin antisera on lysates of testes from wild-type animals and Pif1 $A^{\prime}$ mutants. $\mathbf{d}^{\prime}$ Quantification analyses of Casepase 3 in fig. $4 \mathbf{d}$. **, p $<0.01$, Mann-whitney test, Mann-whitney test. e Real-time PCR analysis of Ark and Dronc transcriptional levels in the testes of wild-type animals and PiffA mutants

individualization in a Drosophila model. Pif $1 A^{1}$ resulted in compromised male fertility but left female fertility unaffected, which is agreed to Miller's communication about the mutation Pif1AZ3-5009 (Miller, K. 2017.3.9, www. flybase.org). We confirmed that loss of Pif1A neither effect on germ cell division nor spermatid elongation, but sperm maturation.

In Drosophila, the Pif1A gene has five annotated transcripts, however, only the Pif1A-RG and Pif1A-RI are highly expressed in the testes of WT animals with Pif1A$R H$ and Pif1A-RJ being barely expressed (Fig. S5). The Drosophila sperm proteome analysis also predicted the Pif1A-RG transcript of Pif1A to exist in sperm ${ }^{58}$. Overexpressing Pif1A-RG and/or Pif1A-RI transgenes driven by a ubiquitously expressed tubulin-gal4 or daughterlessgal4 in the Pif1 $A^{1}$ mutants was not able to rescue the male infertility. One possible explanation is that the overexpression of Pif1A-RG and/or Pif1A-RI, as driven by tubulin-gal4 or daughterless gal4 in Pifl $A^{1}$ mutant background, does not specifically display during spermatogenesis. The Pif1A mutants were frame shift mutants, and the duplication of the Pif1A genome (Pif1A-GFP.FPTB) could partially restore the male reproductive capacity (Fig. 1c). Our consideration raised for this partial rescue is that the insertion position of Pif1A-GFP.FPTB might not be strong enough to produce sufficient Pif1A protein.

Using the Pif1A mutant testis as a spermatogenesis model system to uncover aspects of male infertility, we were able to assess gene function by screening through the whole spermatogenic developmental processes from GSC division to spermatid differentiation, including the spermatid nucleus shaping and the spermatid elongation processes. Without Pif1A, disruption of the IC's synchronic movement occurs where the mutant lacks any $\mathrm{CB}$ and WB and displays disorganized actin cones (Fig. $5 \mathrm{~h}-\mathrm{j}$ ). The elongated spermatids in the mutant seemed eventually undergoing cell death (Fig. S6).

Lipids are essential membrane structural components ${ }^{59}$. Metabolic regulators of lipids such as nes, oys, noa, osbp, and $f a n$ have been reported to play critical roles, particularly during Drosophila sperm individualization ${ }^{29}$. We detected significantly decrease in transcriptional expression of these genes in the Pif1A mutant testes. The phenotype of the Pif1A mutant with defects in individualization and male-sterile was highly reminiscent of the $o s b p$ mutants $^{30}$. These findings suggest that Pif1A might act as a potent regulator to control the lipid metabolism, therefore having an effect on plasma membrane remodeling during sperm individualization.

The nuclear localization of Pif1A in the testis (Fig. 7a, $\mathrm{a}^{\prime)}$ might invite speculation for its roles in cyst cells during spermatogenesis. Cyst cells in flies are analogous to mammalian sertoli cells that provide nutrients and physical support for spermatids and also secrete key regulators involved in spermatogenic development ${ }^{17,60}$. Disruption of such a process is expected to impair spermatogenesis and result in no sperm in the seminal vesicle. In humans, nearly $7 \%$ of men from the worldwide general 


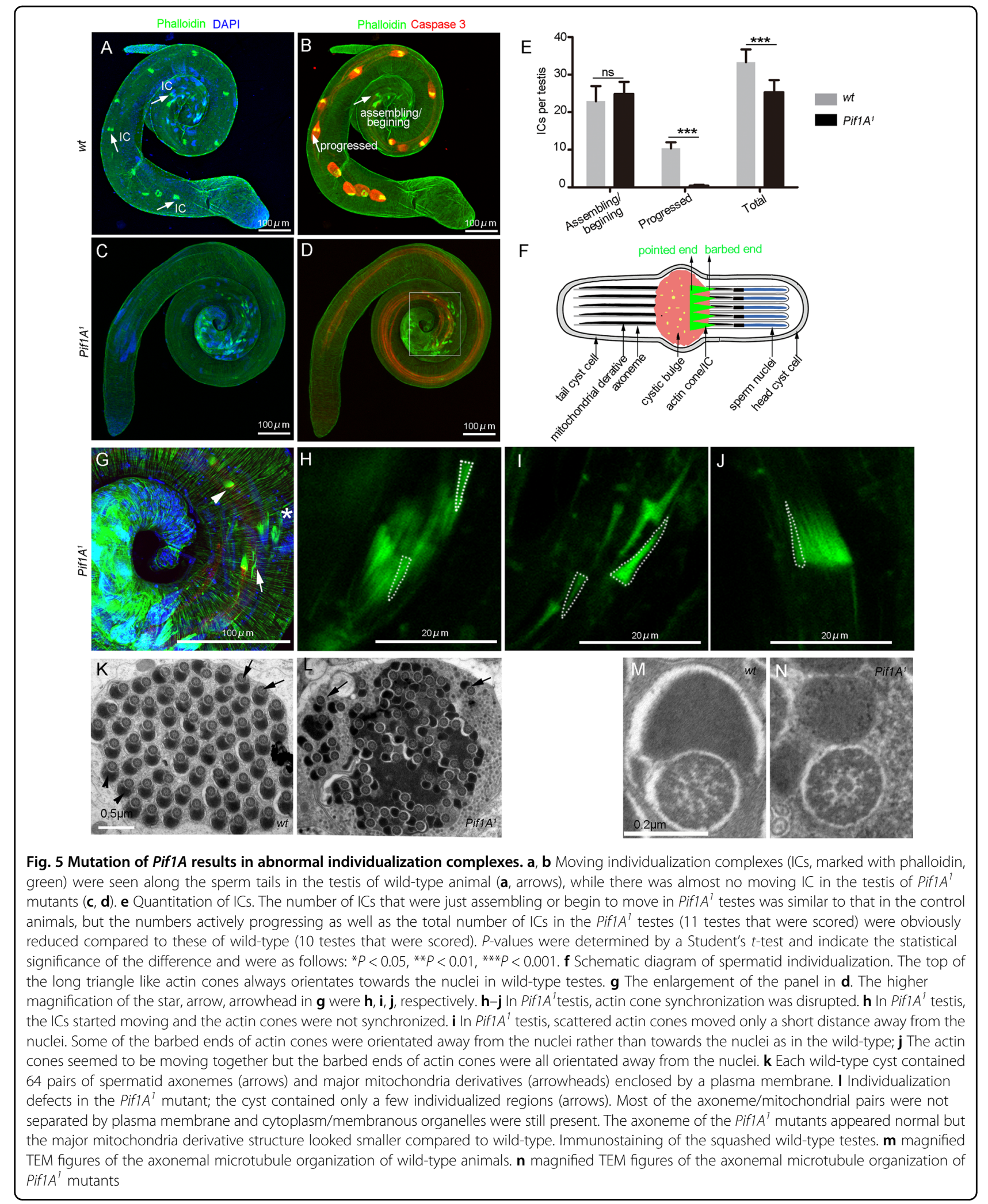




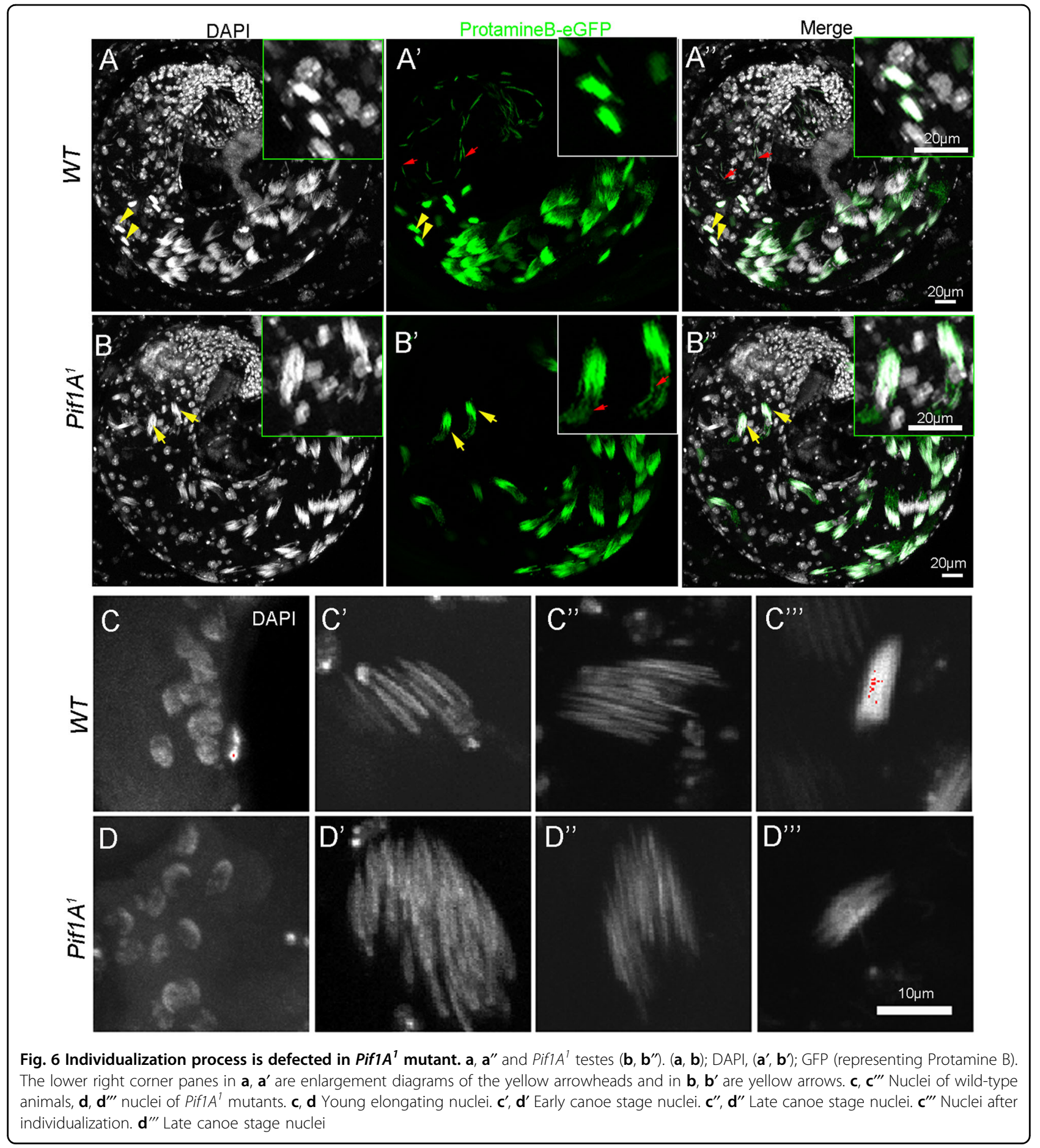

population are infertile. The majority of defects in sperm production are categorized as $\mathrm{NOA}^{61,62}$. Three types of NOA patients all show down-regulation of $\mathrm{CCDC} 157^{13}$ (Fig. 2d). The coiled-coil domains contained in CCDC157 have been reported as common oligomerization domains for a wide range of proteins such as structural proteins, motor proteins and transcription factors $^{63}$. None of these molecular functions has been specifically confirmed for CCDC157. Our data reveals that Pif1A, the homolog of CCDC157, plays a critical role in the individualization process and thus is essential for male fertility. Our study may inform the potential roles of CCDC157 in human spermatogenesis and may provide a new target for the treatment of human male infertility. 

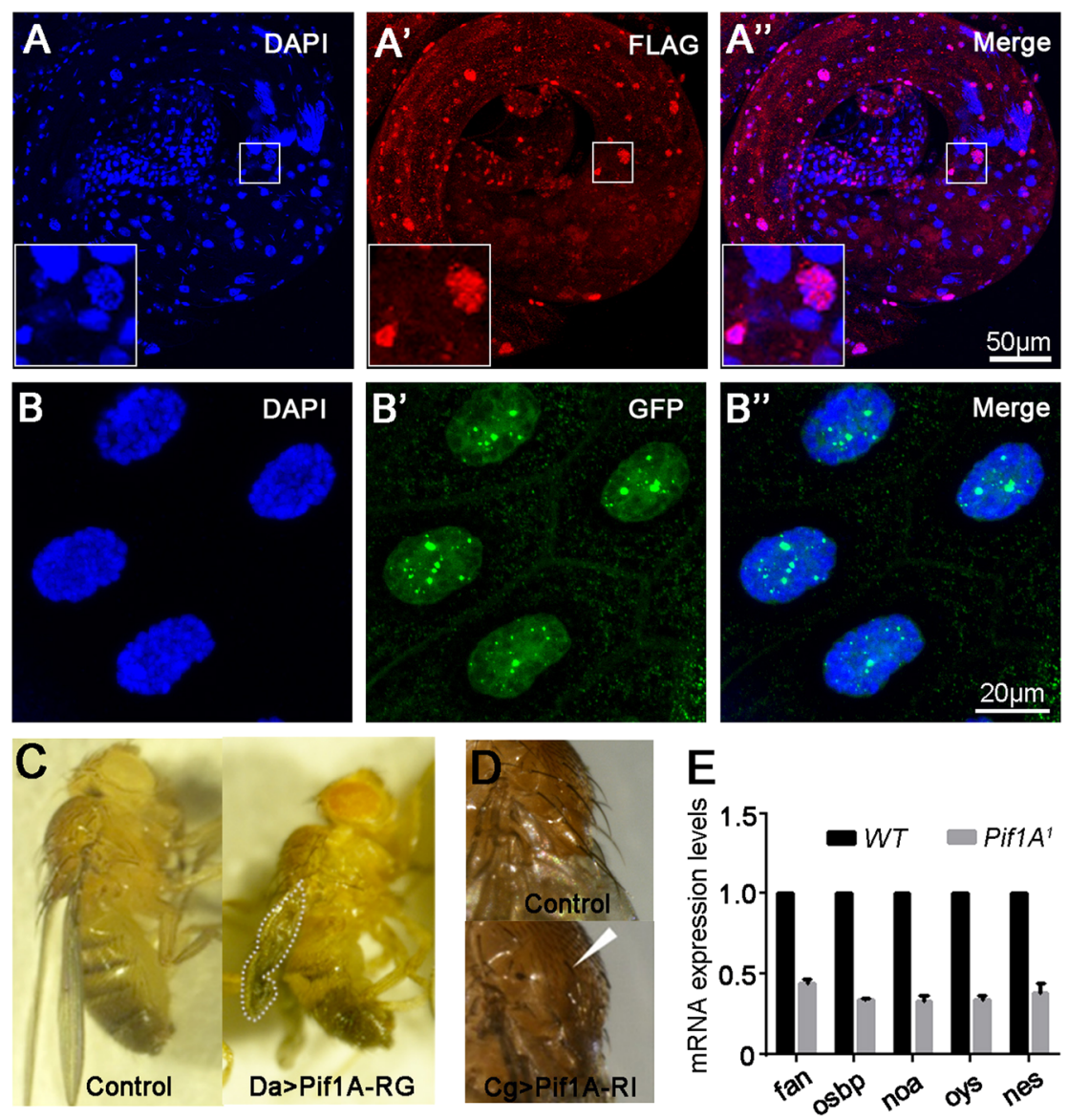

Fig. 7 Pif1A influence lipid metabolism genes. $\mathbf{a}$ - $\mathbf{a}^{\prime \prime}$ The expression of Flag-tagged Pif1A-RI in the testis from BL42670 animals (a'). Nuclei were

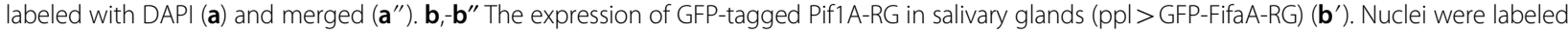
with DAPI (b) and merged $\left(\mathbf{b}^{\prime \prime}\right)$. c Wing expansions after eclosion. White dots indicate the unexpanded wings of ovexpressing Pif1A-RG animals. d Dorsal bristles after eclosion. The white triangle indicates shorter bristles. e RT-PCR amplification of lipid metabolic genes from testes of wild-type (WT) and Pif1A mutants. rp49 was used as a loading control

\section{Materials and methods}

\section{Drosophila stocks and genetics}

Flies were reared on standard cornmeal medium at 25 ${ }^{\circ} \mathrm{C}$. The following strains were used: $\mathrm{w}^{1118}$, tubublin-Gal4, daughterless-Gal4,Cg-Gal4, ppl-Gal4,ß2tubulin-GFP; Bloomington Drosophila Stock Center Df(3R)BSC478/ TM6(BL24982), Dj-GFP (BL5417), Pif1A-GFP.FPTB/Cyo (BL42670), dj-GFP(BL5417), protamine B-eGFP/Cyo (BL58406); bam-Gal4 and tj-Gal4 (kindly provided by Chao Tong); nos-Gal4 and UAS-actin.GFP/CyO; Sb/ TM6B (Tsinghua Fly Center).

To generate the Pif1A mutants, we employed the "CRISPR/Cas9 method", the gRNA was designed to recognize a 19-nt target sequence and act to direct Cas9mediated cleavage of both DNA strands within the target site.

To generate UAS-Pif1A-RI, UAS-Pif1A-RG, and UASeGFP-Pif1A-RG transgenenic flies, we amplified the full length Pif1A cDNA with the primers below and cloned it into the pUAST-attb vector. These constructs were then transformed into VK33 embryos using the standard Pelement-mediated transgenesis protocol.

Pif1A- RG-FCTGCGGCCGCGGCTCGAGATGGCTG

AAAACCAAACCAAAACG

Pif1A- RG-RTCACAAAGATCCTCTAGATCAGAGC

CGGGCATTCTCGGACGG

Pif1A- RI-FCTGCGGCCGCGGCTCGAGATGGGCAA CGAGGAATCCT

Pif1A- RI-RTCACAAAGATCCTCTAGACTATTTCT

TAGCTCTGAACAAG

eGFP-Pif1A- RG-F TTCGTTAACAGATCTGCATGG TGAGCAAGGGCGAGGA

eGFP-Pif1A- RG-R ATCTCGAGCCGCGGCCGCCAC TTGTACAGCTCGTCCATG

\section{Fertility test}

One-day-old males (1 day) from different genotypes were arranged to mate with wild-type 3-5-day-old 
virgin females in small cages (each cage containing 20 males and 40 females) for one day before calculating the embryo hatch rate. Embryos were then collected on new apple juice agar plates for $4 \mathrm{~h}$ and incubated at $25^{\circ} \mathrm{C}$ and $45-70 \%$ humidity for $24 \mathrm{~h}$. Hatch rates were determined from the proportion of hatched eggs to total eggs (200 each time randomly selected from the plates). Under microscopy, one can easily distinguish the hatched embryos showing shriveled egg shells from the unhatched eggs with the appearance of plump grains of white rice. This experiment was repeated three times.

\section{Western blotting}

Protein extracts from testes were prepared by grinding tissue in lysis buffer (1xRIPA buffer: $50 \mathrm{mM}$ Tris- $\mathrm{HCl}, \mathrm{pH}$ 8.0, $150 \mathrm{mM} \mathrm{NaCl}, 1 \%$ IGEPAL CA-630, 0.5\% sodium deoxycholate, $0.1 \%$ SDS) containing the protease inhibitor cocktail (Roche). The lysates were cleared by centrifugation at $14,000 \times g$ for $10 \mathrm{~min}$ at $4{ }^{\circ} \mathrm{C}$. Samples were subjected to sodium dodecyl sulfate polyacrylamide gel electrophoresis (SDS/PAGE) and transferred to polyvinylidene fluoride membrane. Membranes were immunoblotted with the primary antibodies and then probed with the secondary antibodies. Rabbit anti-cleaved Caspase 3 (1:100; Cell Signaling) and mouse anti-tubulin (1:1000; Developmental Studies Hybridoma Bank, E7) were used as the primary antibodies. Blots were treated with the ChemiLucent ECL detection reagents (Millipore) and protein bands were visualized using the Chemiluminescence Imaging System (Clinx Science Instruments, Shanghai, China).

\section{Transmission electron microscopy}

Five days after eclosion the testes were dissected from adult males and prepared for electron microscopy using standard protocols. Thin sections were observed and photographed using a Hitachi H-7650 transmission electron microscope.

\section{Quantitative RT-PCR}

Animal experiments were conducted in accordance with the Guidelines for the Care and Use of Laboratory Animals of Zhejiang University. Total RNA from was extracted using RIzol reagent (Invitrogen) from 5-day-old adult testes. The first-strand cDNA was synthesized from $2 \mu \mathrm{g}$ of total RNA samples using an Invitrogen FirstStrand cDNA synthesis kit. We used Power SYBR Green PCR Master Mix (Applied Biosystems) to conduct RT-PCR with rp49 used as control. Real-time PCR was performed on an ABI7900HT Fast real-time PCR machine. The PCR primers used are provided in below;

rp49-qf GCTAAGCTGTCGCACAAA, rp49-qr TCCG GTGGGCAGCATGTG;
Pif1A-all-qf CAATGCCAGCTCTTGGAACG, Pif1Aall-qr AATCACGACCCTCGAGATC; pif-RA-qF AAATCAAATGGTGAAATTAC,pif-RA-qR GTTGGGTTATCTTGACATCC; pif-RI-qF TCTGGACTGTGTCCATAACC, pif-RI-qR ACAAGGTGGAATTGCTCCTC; pif-RJ-qFAATAATTTCCCCTGTGTTGA, pif-RJ-qR GATTCTCCTCAATAAATTTG; pif-RH-qF GTTGCCCAAGACTCCTCTTA, pif-RH-qR TCTCCTCAATTATTTGCATT; pif-RG-qF CTGTTTACATTACAACATCG, pif-RG-qR TCTCCTCAATCATTAGCTCT; ark-qf GCCTGCAACCGTATCTGAAGCTAA, ark-qr TCTTCAAGTTAAGCCAGAAGATC; dronc-qf GCGTTAGCAAGCTCCGGAATGA, dronc-qr CCTCGGTTGAAACGTGATTGC;

Nessy-qfATTCTGGCAGGTTATCCGGT, NessyqrGGACGTGTAGAAATATCCCA; Oys-qfTTGTCCAAAGAGCCGTCCTG, OysqrCTAGGATGCTTTGGAAGTGC; Noa-qfTCGGCCTAGATTCCAATTGC, NoaqrGGTGATGTGGTGATACCAGT; Osbp-qfGTACGTTCAAATCAATCCCT, OsbpqrCCACTTGACCTCGTTGTCTT;

Fan-qfCTTTGAGGGACCATTCAACC, FanqRCTGCATCTTCCAAAACTCCT;

Ccdc157-qf GGAGGAGAACAAGCGGCTCC, Ccdc157-qr GCCTGTCAACCCTGAGAATG;

L17-qf CGGTATAATGGTGGAGTTG; L17-qr ACCCTTAAGTTCAGCGTTACT.

\section{Microscopy and imaging}

The testes of 5-day adult males were dissected in phosphate-buffered saline (PBS). They were then fixed in PBS containing $4 \%$ formaldehyde for $15 \mathrm{~min}$ at room temperature and washed three times for 15 min each in PBS containing 1\% Triton X-100 (PBT) before being blocked for $1 \mathrm{~h}$ in PBT containing 5\% BSA. The samples were then incubated with primary antibodies (rabbit anticleaved Caspase 3, 1:100, Cell Signaling; Mouse anti-ATP synthase complex $\mathrm{V}$ subunit alpha antibody, Abcam, 1:1000) overnight at $4{ }^{\circ} \mathrm{C}$ followed by four $15 \mathrm{~min}$ washes in PBT. The samples were incubated with secondary antibodies Alexa Fluor 555 -conjugated (1:500, Molecular Probes) for $1 \mathrm{~h}$ at room temperature. Phalloidin staining was performed using Alexa Fluor 488 (Invitrogen) (1:500) for $30 \mathrm{~min}$ at room temperature. 4', 6-diamidino-2phenylindole (DAPI) $(1 \mathrm{~g} / \mathrm{ml}$, Sigma, St. Louis, MO, USA) was added to stain the nuclei. Tissues were mounted and viewed. The slides were observed and photographed using an Olympus FV1000 confocal laser scanning microscope and processed using Adobe Photoshop. To quantify the number of ICs in each testis, a confocal $\mathrm{Z}$ series of the testes were acquired at a step size 
of $1.5 \mathrm{~mm}$. For the measure of nebenkern size, images were processed with ImageJ (National Institutes of Health, Bethesda, MD, USA) to measure the relative areas of nebenkern as an indicator of the size and data were analyzed with a Student's $t$-test. For the white light images of testes, 5-day adult males were dissected in PBS and placed on a glass slide containing a drop of PBS at $25^{\circ} \mathrm{C}$ then obtained using a Nikon SMZ1500 microscope.

For live imaging, the testes of 3 day-old adult males from control animals and Pif $1 A^{1}$ mutants were dissected in Schneider's medium and observed under a Nikon Eclipse 80i fluorescence microscope with a 20 objective lens. Images were acquired at 29.97 frames per second.

\section{Sequence and statistical analyses}

Alignment analysis was performed using https://www. ebi.ac.uk/Tools/msa/clustalo/. Protein structure prediction analysis was performed using SWISS-MODEL (https://swissmodel.expasy.org/) and visualized by UCSF Chimera program. We calculated and generated the plots underlying the differences of CCDC157 mRNA expression levels in nine types of mouse and human tissues respectively, and in 16 patients with NOA compared with four men with normal spermatogenesis by performing the one-way Analysis of variance (ANOVA) by GraphPad Prism 6 (GraphPad Software, Inc., La Jolla, CA, USA). The expression levels of CCDC157 were analyzed by Mann-Whitney test.

\section{In situ hybridization}

Whole-mount adult testis RNA in situ hybridization (ISH) was performed as described ${ }^{64}$. Digoxygenin (dig)labeled RNA probes are in vitro transcribed from a template synthesized by (RT)-PCR; Pif1A probe was generated by using Pif1A-RG mentioned above as the template with primers Pif1A-F, GGCTACACTTCTTCTATACA; Pif1A-R TACGACTCACTATAGGATTTTGTTCTCCA GCGTG. The antisense probe was generated by using primers Control-F TAATACGACTCACTATAGGCTA CACTTCTTCTATACA; Control-R GGATTTTGTTC TCCAGCGTG. The Pif1A probe was the five annotated transcripts common sequence. Detection is through alkaline phosphatase-conjugated anti-dig antibodies followed by a color reaction.

\section{Acknowledgements}

We thank Chris Wood for discussion on the manuscript and the Bloomington Drosophila Stock Center, the Exelixis Collection at the Harvard Medical School and the Tsinghua Fly Center for providing the fly stocks. This study was supported by grants from National Key R\&D Program of China

(2018YFC1004900), National Natural Science Foundation of China (31801230),

China Postdoctoral Science Foundation (2018M632452).

\section{Author details}

'Division of Human Reproduction and Developmental Genetics, the Women's Hospital, Zhejiang University School of Medicine, Yuhangtang Road 866, 310012 Hangzhou, Zhejiang, China. ${ }^{2}$ Institute of Genetics and Department of
Genetics, Zhejiang University School of Medicine, Yuhangtang Road 866, 310012 Hangzhou, Zhejiang, China. ${ }^{3}$ Joint Institute of Genetics and Genomic Medicine between Zhejiang University and University of Toronto, Zhejiang University, Hangzhou, Zhejiang, China

\section{Authors' contributions}

Y.X., C.L, X.Y., and H.Z., designed the experiments, interpreted the data, wrote the manuscript; X.Y., H.Z., Y.S., P.G., W.G., and X.Z. conducted the Pif1A experiments and analyzed the data. C.L., X.Y., and Q.Z. conducted the mammalian CCDC157 experiments and analyzed the data. All authors contributed helpful suggestions for this manuscript.

\section{Conflict of interest}

The authors declare that they have no conflict of interest.

\section{Publisher's note}

Springer Nature remains neutral with regard to jurisdictional claims in published maps and institutional affiliations.

Supplementary Information accompanies this paper at (https://doi.org/ 10.1038/s41419-019-1398-3).

Received: 31 July 2018 Revised: 24 January 2019 Accepted: 25 January 2019 Published online: 11 February 2019

\section{References}

1. Ramm, S. A., Schärer, L., Ehmcke, J. \& Wistuba, J. Sperm competition and the evolution of spermatogenesis. MHR: Basic Sci. Reprod. Med. 20, 1169-1179 (2014).

2. Aston, K. I. Genetic susceptibility to male infertility: news from genome-wide association studies. Andrology 2, 315-321 (2014).

3. Fuller, M. Spermatogenesis. The Development of Drosophila melanogaster. (eds.) M. Bate and A. Martinez-Arias. (Cold Spring Harbor, New York: Cold Spring Harbor Press) 1, 71-147 (1993).

4. Wakimoto, B. T., Lindsley, D. L. \& Herrera, C. Toward a comprehensive genetic analysis of male fertility in Drosophila melanogaster. Genetics 167, 207-216 (2004).

5. Yu, J. et al. Protein synthesis and degradation are essential to regulate germline stem cell homeostasis in Drosophila testes. Development 143 2930-2945 (2016)

6. Fabian, L. \& Brill, J. A. Drosophila spermiogenesis: big things come from little packages. Spermatogenesis 2, 197-212 (2012).

7. Siddall, N. A. \& Hime, G. R. A Drosophila toolkit for defining gene function in spermatogenesis. Reproduction 153, R121-R132 (2017).

8. Lu, C., Kim, J., Fau-Fuller, M. T. \& Fuller, M. T. The polyubiquitin gene Ubi-p63E is essential for male meiotic cell cycle progression and germ cell differentiation in Drosophila. Development 140, 3522-3531 (2013).

9. Huh, J. R. et al. Multiple apoptotic caspase cascades are required in nonapoptotic roles for Drosophila spermatid individualization. PLOS Biol. 2, E15 (2004).

10. Bassett, A. \& Liu, J.-L. CRISPR/Cas9 mediated genome engineering in Drosophila. Methods 69, 128-136 (2014).

11. Brand, A. H. \& Perrimon, N. Targeted gene expression as a means of altering cell fates and generating dominant phenotypes. Development 118, 401-415 (1993).

12. Rascle, A., Stowers, R. S., Garza, D., Lepesant, J. -A. \& Hogness, D. S. L63, the Drosophila PFTAIRE, interacts with two novel proteins unrelated to cyclins. Mech. Dev. 120, 617-628 (2003).

13. Reinke, A. W., Baek, J., Ashenberg, O.\& Keating, A. E. Networks of bZIP proteinprotein interactions diversified over a billion years of evolution. Science $\mathbf{3 4 0}$, 730-734 (2013).

14. Wang, M. et al. Single-cell RNA sequencing analysis reveals sequential cell fate transition during human spermatogenesis. Cell Stem Cell 23(1875-9777 (Electronic), 599-614 (2018).

15. Tokuyasu, K, Peacock, W. \& Hardy, R. Dynamics of spermiogenesis in Drosophila melanogaster. Cell Tissue Res. 127, 492-525 (1972).

16. de Cuevas, M. \& Matunis, E. L. The stem cell niche: lessons from the Drosophila testis. Development 138, 2861-2869 (2011). 
17. Zoller, R. \& Schulz, C. The Drosophila cyst stem cell lineage: Partners behind the scenes? Spermatogenesis 2, 145-157 (2012).

18. Fabrizio, J. J., Hime, G., Lemmon, S. K. \& Bazinet, C. Genetic dissection of sperm individualization in Drosophila melanogaster. Development 125, 1833-1843 (1998).

19. Tokuyasu, K. T. Dynamics of spermiogenesis in Drosophila melanogaster. VI. Significance of "onion" nebenkern formation. J. Ultrastruct. Res. 53, 93-112 (1975).

20. Noguchi, T. \& Miller, K. G. A role for actin dynamics in individualization during spermatogenesis in Drosophila melanogaster. Development 130, 1805-1816 (2003).

21. Arama, E., Agapite, J. \& Steller, H. Caspase activity and a specific cytochrome C are required for sperm differentiation in Drosophila. Dev. Cell 4, 687-697 (2003).

22. Rotkopf, S. et al. The WASp-based actin polymerization machinery is required in somatic support cells for spermatid maturation and release. Development 138, 2729-2739 (2011).

23. Cooper, J. A., Wear, M. A. \& Weaver, A. M. Arp2/3 complex: Advances on the inner workings of a molecular machine. Cell 107, 703-705 (2001).

24. Mermall, $\mathrm{V}$. et al. Drosophila myosin $\mathrm{V}$ is required for larval development and spermatid individualization. Dev. Biol. 286, 238-255 (2005).

25. Rogat, A. D. \& Miller, K. G. A role for myosin VI in actin dynamics at sites of membrane remodeling during Drosophila spermatogenesis. J. Cell Sci. 115, 4855-4865 (2002).

26. Hicks, J. L. et al. Class VI unconventional myosin is required for spermatogenesis in Drosophila. Mol. Biol. Cell 10, 4341-4353 (1999).

27. Cooper, J. A. \& Schafer, D. A. Control of actin assembly and disassembly at filament ends. Curr. Opin. Cell Biol. 12, 97-103 (2000).

28. Noguchi, T., Lenartowska, M., Miller, K. G. \& Myosin, V. I. stabilizes an actin network during Drosophila spermatid individualization. Mol. Biol. Cell 17, 2559-2571 (2006)

29. Wang, C. \& Huang, X. Lipid metabolism and Drosophila sperm development. Sci. China Life Sci. 55, 35-40 (2012).

30. Ma, Z., Liu, Z. \& Huang, X. OSBP- and FAN-mediated sterol requirement for spermatogenesis in Drosophila. Development 137, 3775-3784 (2010).

31. Jung, A., Hollmann, M. \& Schafer, M. A. The fatty acid elongase NOA is necessary for viability and has a somatic role in Drosophila sperm development. J. Cell Sci. 120(Pt 16), 2924-2934 (2007).

32. Wang, C., Ma, Z., Scott, M. P. \& Huang, X. The cholesterol trafficking protein NPC1 is required for Drosophila spermatogenesis. Dev. Biol. 351, 146-155 (2011).

33. Fagerberg, L. et al. Analysis of the human tissue-specific expression by genome-wide integration of transcriptomics and antibody-based proteomics. Mol. Cell. Proteom.: MCP 13, 397-406 (2014).

34. Bunt, S. M., Monk, A. C., Siddall, N. A., Johnston, N. L. \& Hime, G. R. GAL4 enhancer traps that can be used to drive gene expression in developing Drosophila spermatocytes. Genesis 50, 914-920 (2012).

35. Shivdasani, A. A. \& Ingham, P. W. Regulation of stem cell maintenance and transit amplifying cell proliferation by TGF- $\beta$ signaling in Drosophila spermatogenesis. Curr. Biol. 13, 2065-2072 (2003).

36. Mikhaylova, L. M. \& Nurminsky, D. I. Lack of global meiotic sex chromosome inactivation, and paucity of tissue-specific gene expression on the Drosophila X chromosome. BMC Biol. 9, 29 (2011).

37. Texada, M. J., Simonette, R. A., Johnson, C. B., Deery, W. J. \& Beckingham, K. M. Yuri gagarin is required for actin, tubulin and basal body functions in Drosophila spermatogenesis. J. Cell Sci. 121, 1926-1936 (2008).

38. Gao, Z., Ruden, D. M. \& Lu, X. PKD2 cation channel is required for directional sperm movement and male fertility. Curr. Biol. 13, 2175-2178 (2003).

39. Noguchi, T., Lenartowska, M., Rogat, A. D., Frank, D. J. \& Miller, K. G. Proper cellular reorganization during Drosophila spermatid individualization depends on actin structures composed of two domains, bundles and meshwork, that are differentially regulated and have different functions. Mol. Biol. Cell 19, 2363-2372 (2008).

40. Frank, D. J., Noguchi, T. \& Miller, K. G. Myosin VI: a structural role in actin organization important for protein and organelle localization and trafficking. Curr. Opin. Cell Biol. 16, 189-194 (2004).
41. Couderc, J. L., Richard, G., Vachias, C. \& Mirouse, V. Drosophila LKB1 is required for the assembly of the polarized actin structure that allows spermatid individualization. PLOS ONE 12, e0182279 (2017).

42. Basu, J. \& Li, Z. The Des-1 protein, required for central spindle assembly and cytokinesis, is associated with mitochondria along the meiotic spindle apparatus and with the contractile ring during male meiosis in Drosophila melanogaster. Mol. General. Genet. MGG 259, 664-673 (1998).

43. Clark, I. E. et al. Drosophila pink1 is required for mitochondrial function and interacts genetically with parkin. Nature 441, 1162 (2006).

44. Oliva, M., De Pinto, V., Barsanti, P. \& Caggese, C. A genetic analysis of the porin gene encoding a voltage-dependent anion channel protein in Drosophila melanogaster. Mol. Genet. Genom. 267, 746-756 (2002).

45. Volpi, S., Bongiorni, S., Fabbretti, F., Wakimoto, B. T. \& Prantera, G. Drosophila rae1 is required for male meiosis and spermatogenesis. J. Cell Sci. 126, 3541 (2013).

46. Gatt, M. K. \& Glover, D. M. The Drosophila phosphatidylinositol transfer protein encoded by vibrator is essential to maintain cleavage-furrow ingression in cytokinesis. J. Cell Sci. 119, 2225-2235 (2006).

47. Bolobolova, E. U., Yudina, O. S. \& Dorogova, N. V. Drosophila tumor suppressor merlin is essential for mitochondria morphogenesis during spermatogenesis in Drosophila melanogaster. Cell Tissue Biol. 5, 136-143 (2011).

48. Dorogova, N. V. et al. The role of Drosophila Merlin in spermatogenesis. BMC Cell. Biol. 9,1 (2008).

49. Jayaramaiah Raja, S. \& Renkawitz-Pohl, R. Replacement by Drosophila melanogaster protamines and Mst77F of histones during chromatin condensation in late spermatids and role of sesame in the removal of these proteins from the male pronucleus. Mol. Cell. Biol. 25, 6165-6177 (2005).

50. Rathke, C. et al. Transition from a nucleosome-based to a protamine-based chromatin configuration during spermiogenesis in Drosophila. J. Cell Sci. 120 (Pt 9), 1689-1700 (2007).

51. Ghosh-Roy, A., Kulkarni, M., Kumar, V., Shirolikar, S. \& Ray, K. Cytoplasmic dynein-dynactin complex is required for spermatid growth but not axoneme assembly in Drosophila. Mol. Biol. Cell 15, 2470-2483 (2004).

52. Ghosh-Roy, A., Desai, B. S. \& Ray, K. Dynein light chain 1 regulates dynaminmediated F-actin assembly during sperm individualization in Drosophila. Mol. Biol. Cell 16, 3107-3116 (2005).

53. $\mathrm{Wu}, \mathrm{C}$. - H. et al. Knockdown of Dynamitin in testes significantly decreased male fertility in Drosophila melanogaster. Dev. Biol. 420, 79-89 (2016).

54. Sitaram, P., Anderson, M. A., Jodoin, J. N., Lee, E. \& Lee, L. A. Regulation of dynein localization and centrosome positioning by Lis-1 and asunder during Drosophila spermatogenesis. Development 139, 2945-2954 (2012).

55. Kracklauer, M. P. et al. The Drosophila SUN protein Spag4 cooperates with the coiled-coil protein Yuri Gagarin to maintain association of the basal body and spermatid nucleus. J. Cell Sci. 123, 2763-2772 (2010).

56. Steinhauer, J. Separating from the pack: molecular mechanisms of Drosophila spermatid individualization. Spermatogenesis 5, e1041345 (2015).

57. Schafer, D. A. \& Cooper, J. A. Control of actin assembly at filament ends. Annu. Rev. Cell. Dev. Biol. 11, 497-518 (1995).

58. Wasbrough, E. R. et al. The Drosophila melanogaster sperm proteome-\| (DmSP-II). J. Proteom. 73, 2171-2185 (2010).

59. Yeagle, P. L. Lipid regulation of cell membrane structure and function. FASEB J. 3, 1833-1842 (1989).

60. Griswold, M. The Central Role of Sertoli Cells in Spermatogenesis, Seminars in Cell \& Developmental Biology. 9, 411-416 (1998).

61. Krausz, C. Male infertility: Pathogenesis and clinical diagnosis. Best. Pract. Res. Clin. Endocrinol. Metab. 25, 271-285 (2011).

62. Hull, M. G. et al. Population study of causes, treatment, and outcome of infertility. Br. Med. J. 291, 1693-1697 (1985).

63. Nooren, I. M., Kaptein, R., Sauer, R. T. \& Boelens, R. The tetramerization domain of the Mnt repressor consists of two right-handed coiled coils. Nat. Struct. Biol. 6, 755-759 (1999).

64. Morris, C. A., Benson, E. \& White-Cooper, H. Determination of gene expression patterns using in situ hybridization to Drosophila testes. Nat. Protoc. 4, 1807 (2009). 